\title{
Long-term depletion of cereblon induces mitochondrial dysfunction in cancer cells
}

\author{
Seulki Park ${ }^{1,2}$, Kidae Kim ${ }^{3}$, Keeok Haam ${ }^{4}$, Hyun Seung Ban ${ }^{4}$,Jung-Ae Kim ${ }^{2,5}$, Byoung Chul Park ${ }^{1,6}$, Sung Goo Park ${ }^{1,2, *}$, \\ Sunhong Kim, ${ }^{7, *}$ Jeong-Hoon Kim ${ }^{1,2, *}$ \\ ${ }^{1}$ Disease Target Structure Research Center, Korea Research Institute of Bioscience and Biotechnology (KRIBB), Daejeon 34141, \\ ${ }^{2}$ Department of Functional Genomics, KRIBB School of Bioscience, Korea University of Science and Technology, Daejeon 34113, \\ ${ }^{3}$ Division of Biomedical Informatics, Center for Genome Science, National Institute of Health, KCDC, Cheongju 28159, ${ }^{4}$ Biotherapeutics \\ Translational Research Center, Korea Research Institute of Bioscience and Biotechnology, Daejeon 34141, ${ }^{5}$ Personalized Genomic Medicine \\ Research Center, Korea Research Institute of Bioscience and Biotechnology, Daejeon 34141, ${ }^{6}$ Department of Proteome Structural Biology, \\ KRIBB School of Bioscience, Korea University of Science and Technology, Daejeon 34113, ${ }^{7}$ Drug Discovery Center, LG Chem, Seoul 07796, Korea
}

\begin{abstract}
Cereblon (CRBN) is a multi-functional protein that acts as a substrate receptor of the E3 ligase complex and a molecular chaperone. While CRBN is proposed to function in mitochondria, its specific roles are yet to be established. Here, we showed that knockdown of CRBN triggers oxidative stress and calcium overload in mitochondria, leading to disruption of mitochondrial membrane potential. Notably, long-term CRBN depletion using PROteolysis TArgeting Chimera (PROTAC) induced irreversible mitochondrial dysfunction, resulting in cell death. Our collective findings indicate that CRBN is required for mitochondrial homeostasis in cells. [BMB Reports 2021; 54(6): 305-310]
\end{abstract}

\section{INTRODUCTION}

The double-membraned mitochondrion possesses pleotropic functions, including regulation of calcium flux, programmed cell death and adenosine triphosphate (ATP) production, in all eukaryotic cells (1). Among these, the paramount role of mitochondria is ATP production through oxidative phosphorylation (OXPHOS) (2). During this process, reactive oxygen species (ROS) are generated as by-products in the electron transport chain on the inner mitochondrial membrane (3). Mitochondrial ROS levels are also significantly increased upon exposure of cells to environmental stress conditions, such as heat shock and xenotoxic exposure (4). Exces-

*Corresponding authors. Sung Goo Park, Tel: +82-42-860-4262; Fax: +82-42-860-4598; E-mail: sgpark@kribb.re.kr; Sunhong Kim, Tel: +82-2-6987-5505; Fax: +82-2-6987-4844; E-mail: skimbio@lgchem. com; Jeong-Hoon Kim, Tel: +82-42-860-4264; Fax: +82-42-860-4598; E-mail: jhoonkim@kribb.-re.kr

https://doi.org/10.5483/BMBRep.2021.54.6.218

Received 5 October 2020, Revised 17 November 2020, Accepted 30 December 2020

Keywords: Calcium overload, Cell death, CRBN, Mitochondrial dysfunction, ROS sive generated ROS induce oxidative damage to mitochondrial DNA and proteins (5). Accumulating oxidative damage leads to mitochondrial dysfunction, in turn, accelerating ROS generation in mitochondria through mitochondrial permeability transition pore (mPTP) opening and loss of mitochondrial proteins (6). As a safeguard against ROS-mediated oxidative damage, mitochondria have a quality control system for removal of oxidized proteins and $\operatorname{ROS}(7,8)$. Mitochondrial Lon protease (Lonp1) is a major regulator of mitochondrial homeostasis. Lonp1 is involved in protein quality control via proteolytic processing of misfolded and damaged proteins in mitochondria (9). Dysregulation of Lonp1 and excessive ROS accumulation are associated with aging, infertility and tumorigenesis (10).

Cereblon (CRBN), a substrate receptor of the E3 ligase complex, contains Lon, a conserved $\mathrm{N}$-terminal domain derived from Lon protease (11). Overexpression of mitochondrial CRBN effectively protects neuroblastoma cells from ROS-induced cell death through reducing oxidative damage to mitochondrial proteins (11), supporting a contributory role in maintenance of mitochondrial homeostasis similar to that of Lon protease. On the other hand, CRBN has been shown to regulate bactericidal activity through modulating mitochondrial ROS. Upon Toll-like receptor 4 (TLR4) stimulation, CRBN suppresses the ubiquitination of Evolutionarily Conserved Signaling Intermediate in Toll pathway (ECSIT) via disruption of ECSIT-TNF Receptor Associated Factor 6 (TRAF6) interactions in monocyte cells (12). These findings suggest that CRBN can control mitochondrial homeostasis through regulating ECSIT, which is essential for stabilization of mitochondrial complex I and ROS. Furthermore, CRBN binds p53 and hinders its interactions with the anti-apoptotic mitochondrial protein B-cell lymphoma 2/B-cell lymphoma-extra Large (Bcl-2/Bcl-xL) (13). Overall, the above results support the theory that $\mathrm{CRBN}$ prevents oxidative damage-induced cell death through facilitating maintenance of mitochondrial homeostasis. However, the mitochondrial functions of CRBN in cancer cells remain unknown.

In this study, we have demonstrated protective roles of mitochondrial CRBN against ROS. Knockdown of CRBN triggered 
mitochondrial membrane potential disruption and calcium overload. Intriguingly, long-term depletion of CRBN via treatment with CRBN PROteolysis TArgeting Chimera (PROTAC) led to irreversible mitochondrial malfunction and apoptotic cell death. Based on the collective findings, we conclude that CRBN plays an essential role in cancer cell survival via maintaining mitochondrial homeostasis.

\section{RESULTS}

\section{Knockdown of cereblon increases oxidative stress and disrupts mitochondrial membrane potential}

To explore the potential roles of CRBN in mitochondria, we induced CRBN knockdown using specific short hairpin RNA (shRNA) in a HepG2 cell line (shCRBN). Quantitative real-time PCR (qRT-PCR) and western blot analyses confirmed decreased mRNA and protein levels of CRBN in shCRBN cells, respectively (Fig. 1A, Supplementary Fig. 1A, B). Clone 5 of shRNA (shCRBN) was mainly used for subsequent experiments. Next, we examined whether CRBN knockdown affects ROS production. For quantitative measurement of oxidative stress, cells were stained with two different ROS indicators. The fluorescence intensity of both indicators was significantly increased in shCRBN cells compared with shControl cells (Fig. 1B, C).

Excessive levels of ROS can disrupt mitochondrial membrane potential, which is essential for ATP generation (6). Accordingly, we examined the mitochondrial membrane potential in shCRBN cells using the membrane-permeable JC-1 dye. Consistent with previous findings (14), hydrogen peroxide $\left(\mathrm{H}_{2} \mathrm{O}_{2}\right)$-treated cells mainly exhibited green fluorescence. Similarly, shCRBN cells displayed higher levels of green fluorescence compared with shControl cells (Fig. 1D, Supplementary Fig. 1C and 4D), clearly indicating that CRBN knockdown impairs mitochondrial membrane potential. However, CRBN knockdown effect on superoxide production and mitochondrial membrane potential in THLE-3 cells, phenotypic characteristics like normal adult liver epithelial cells, showed no significant changes (Supplementary Fig. 2A, $B)$. We further investigated mitochondrial respiration activity in shCRBN cells by measuring total ATP production rate and mitochondrial oxygen consumption rate (OCR). The total ATP production rate, including glycolytic (glycoATP) and mitochondrial ATP (mitoATP), was decreased in shCRBN cells (Fig. 1E). Moreover, both basal and ATP-linked respiration rates were decreased in shCRBN cells (Fig. 1F, G). The collective data support a critical role of CRBN in maintenance of mitochondrial homeostasis and cellular metabolism.

\section{CRBN knockdown induces calcium overload in mitochondria}

One of the main functions of mitochondria is regulation of calcium homeostasis (15). Excessive ROS causes loss of mitochondrial membrane potential, in turn, leading to increased calcium influx. Conversely, calcium overload promotes mitochondrial ROS generation (16). To determine whether CRBN knockdown affects
A
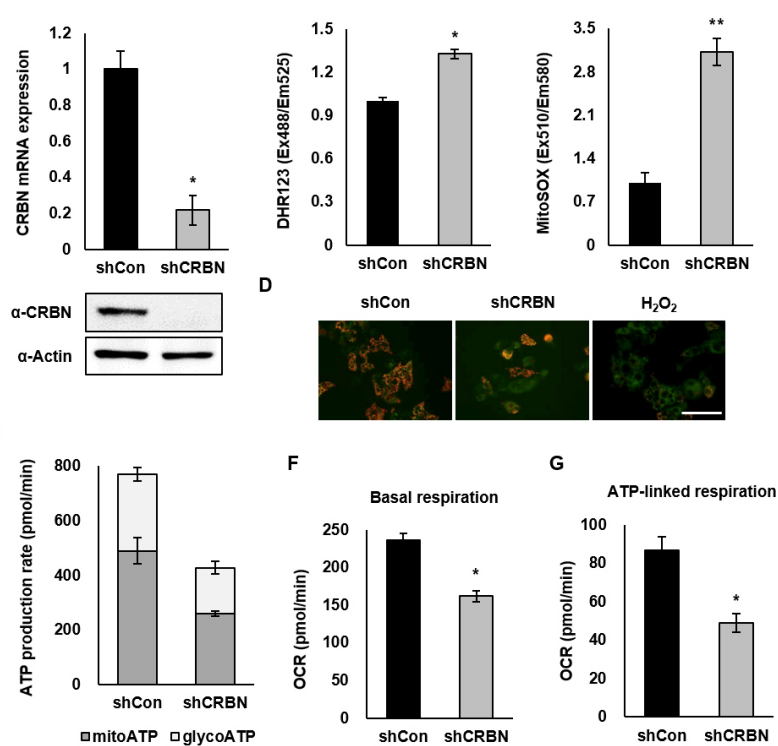

Fig. 1. CRBN knockdown leads to excessive ROS production and mitochondrial dysfunction. (A) Levels of CRBN mRNA (top) and protein (bottom) were examined via qRT-PCR and western blot, respectively, in shControl (shCon) and stable CRBN-depleted (shCRBN) HepG2 cells. (B, C) Fluorescence staining of each ROS species. shControl and shCRBN HepG2 cells were stained with (B) DHR123, an indicator of intracellular hydrogen peroxide, or (C) MitoSOX Red, an indicator of mitochondrion-derived superoxide. (D) To monitor mitochondrial membrane potential, shControl HepG2 cells treated with or without $\mathrm{H}_{2} \mathrm{O}_{2}$ $100 \mu \mathrm{M}$ and shCRBN HepG2 cells were stained using JC-1 fluorescent dye and images visualized under a fluorescence microscope. Representative images are shown. Original magnification $(\times 200)$; Scale bar: $50 \mu \mathrm{m}$. (E) Quantitative measurement of ATP production using the Seahorse Extracellular Flux Analyzer. (F) Basal respiration was calculated as the mean value of the first three baseline readings. (G) ATP-linked respiration OCR was measured based on the subtracted value of the mean of three readings following oligomycin $(1.5 \mu \mathrm{M})$ treatment from basal respiration. All results are presented as mean \pm S.D. of at least three independent experiments. P-values were calculated using unpaired Student's t-test. ${ }^{*} \mathrm{P}<0.05$ and $* * \mathrm{P}<0.01$ vs control.

mitochondrial calcium levels, we used Rhod2-AM, a calcium sensor that can penetrate the mitochondrial membrane (17). To this end, we additionally established shCRBN cells using the HeLa cell line. HepG2 and HeLa cells were stained with Rhod2AM, MitoTracker, and Hoechst, followed by imaging using confocal microscopy. Extracellular uridine triphosphate (UTP) treatment is reported to activate intracellular calcium signaling through the UTP-sensitive receptor (18). Confocal microscopy analysis revealed a significant increase in mitochondrial calcium levels at 120 seconds after UTP treatment in shCRBN, but not shControl cells (Fig. 2). Calcium levels were restored to normal in shControl cells but remained high in shCRBN cells at 240 seconds after UTP treatment (Fig. 2). However, the cytosolic calcium response to UTP treatment was not altered in shCRBN relative to shControl cells (Supplementary Fig. 3A, B). 
A
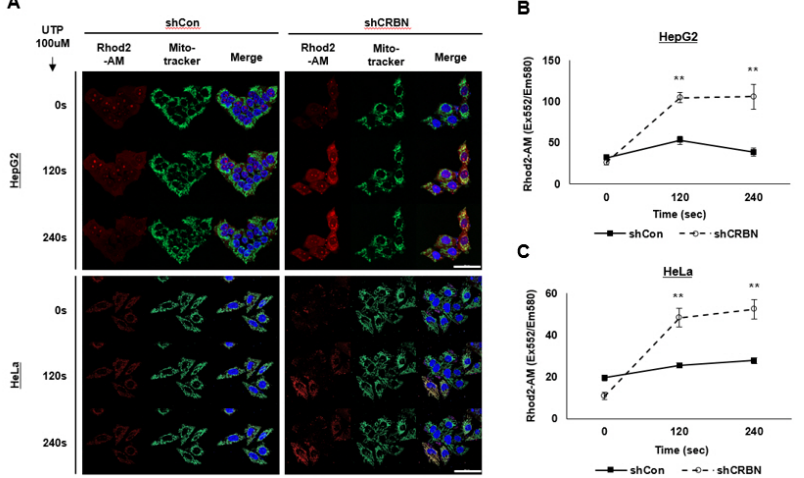

Fig. 2. CRBN knockdown induces mitochondrial calcium overload. (A) Analysis of calcium flux in mitochondria using Rhod2-AM fluorescent dye. MitoTracker green and Hoechst were employed as mitochondrial and nuclear indicators, respectively. All cells were stained with indicators and imaged under a confocal microscope for 240 seconds following UTP treatment $(100 \mu \mathrm{M})$. Original magnification (X630) Scale bar: $50 \mu \mathrm{m}$. (B, C) Quantitative analysis of mitochondrial calcium flux in (B) shControl and shCRBN HepG2 cells and (C) shControl and shCRBN HeLa cells. The $\mathrm{Y}$ axis represents fluorescence intensity of Rhod2-AM analyzed using Zen2 (blue edition) program. P-values were calculated using unpaired Student's t-test. $* \mathrm{P}<0.05$ and $* * \mathrm{P}<0.01$ vs control.

Our results indicate that CRBN affects mitochondrial calcium flux through regulating ROS production and mitochondrial potential.

\section{Long-term depletion of CRBN induces irreversible mitochondrial dysfunction}

Next, we examined whether mitochondrial dysfunction in shCRBN cells could be rescued by exogenous expression of CRBN. To neutralize the effects of shRNA, we generated a plasmid expressing shRNA-resistant mutant CRBN, CRBN-Flag (R). Expression of CRBN-FLAG (R) was validated via western blot (Fig. 3A). Interestingly, CRBN-Flag $(R)$ expression rescued elevated mitochondrial superoxide to control levels but not impaired mitochondrial membrane potential in shCRBN cells (Fig. 3B, C). In addition, CRBN knockdown using small interfering RNA (siRNA) did not induce changes in the hydrogen peroxide and superoxide levels (Supplementary Fig. 4A-C). The results suggest that a prolonged period of CRBN deficiency could lead to irreversible ROS-induced mitochondrial damage. To ascertain the effects of long-term CRBN deficiency on mitochondrial function, experiments were performed with a previously reported CRBN degrader, TD-165 (19). As expected, TD-165 treatment induced efficient degradation of CRBN (Fig. 3D). Consistent with the results obtained with shCRBN cells, hydrogen peroxide production was increased in cells treated with TD-165 for 3 and 12 days (Fig. $3 \mathrm{E})$. However, a significant increase in superoxide production was observed in cells treated for 12 days and not those subjected to 3 days of treatment (Fig. 3F), indicating that superoxide accumulation is promoted only following long-term depletion of CRBN. In keeping with this finding, mitochondrial membrane
A
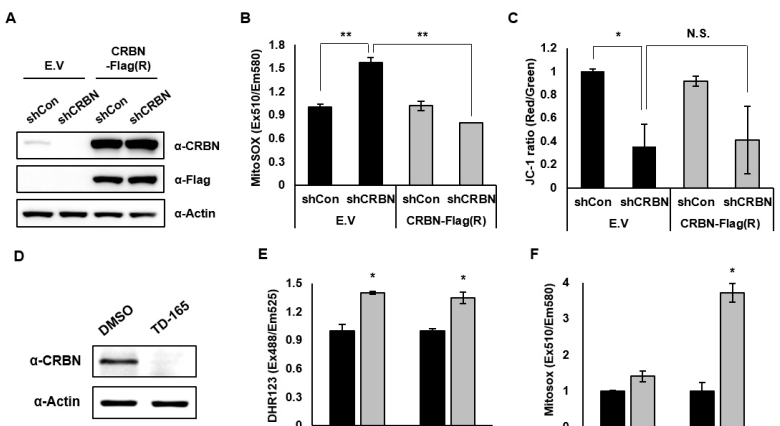

$$
\text { E }
$$
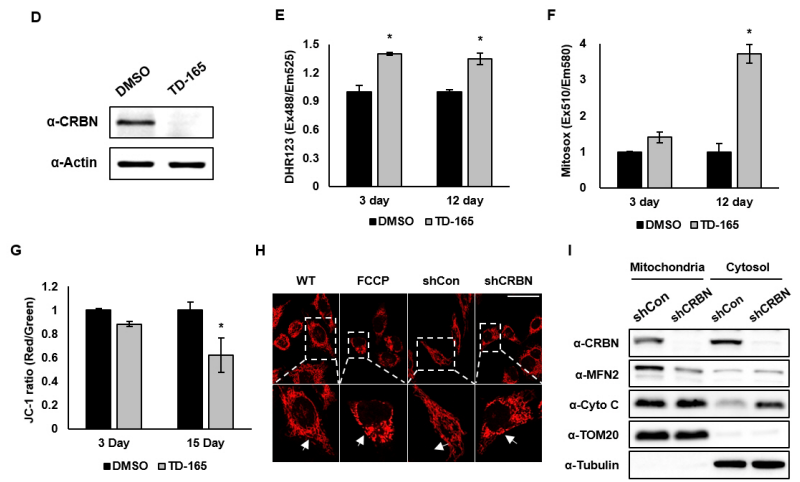

Fig. 3. Long-term depletion of CRBN promotes mitochondrial ROS accumulation and irreversible mitochondrial damage. (A) Exogenous CRBN expression in shControl and shCRBN cells determined via western blot. (B) Quantitative analysis of mitochondrial superoxide production using MitoSOX Red fluorescent dye. (C) Quantitative analysis of mitochondrial membrane potential via flow cytometry in CRBN-overexpressing cells. (D) Western blot analysis of CRBN levels in TD-165-treated HepG2 cells. (E) Quantitation of intracellular hydrogen peroxide production after TD-165 treatment $(1 \mu \mathrm{M})$ for 3 and 12 days. (F) Quantitation of mitochondrion-derived superoxide production after TD-165 treatment $(1 \mu \mathrm{M})$ for 3 and 12 days. (G) Quantitative analysis of mitochondrial membrane potential via flow cytometry. $(\mathrm{H})$ Immunofluorescence staining of shControl and shCRBN HeLa cells using TOM20 antibody. Images were visualized via confocal microscopy. Arrows indicate changes in the shape of mitochondria. Original magnification (X630) Scale bar: $50 \mu \mathrm{m}$. (I) Western blot analysis of CRBN and mitochondria-related protein expression after isolation of mitochondrial and cytosol extracts from CRBN-depleted HepG2 cells. TOM20 and alpha-tubulin were used as the controls for mitochondria and cytosol, respectively. Results are presented as mean \pm S.D. of at least three independent experiments. $\mathrm{P}$-values were calculated using unpaired student's t-test. ${ }^{*} \mathrm{P}<0.05$ and $* * \mathrm{P}<0.01$ vs control.

potential was decreased following TD-165 treatment in a timedependent manner (Fig. 3G). To exclude off-target effects on cytotoxicity by TD-165, we treated TD-165 for 12 days in shControl and shCRBN cells. Long-term treatment of TD-165 in shCRBN cells showed no additional effect on cell viability (Supplementary Fig. 5A, B). Moreover, immunostaining analysis of translocase of outer membrane 20 (TOM20), a mitochondrial outer membrane receptor responsible for recognition and translocation of cytosol-synthesized proteins (20), showed that mitochondria in shCRBN cells were shortened and highly aggregated, similar to those treated with the mitochondrial uncoupler trifluoromethoxy carbonylcyanide phenylhydrazone (FCCP) (Fig. 3H) (21). A cellular compartmentation experiment revealed decreased levels of the mitochondrial fusion protein Mitofusin 2 (MFN2) (22) in mitochondrial fractions and increased cytochrome C (cyto C), 
a hallmark of apoptosis, in cytosolic fractions of shCRBN cells (Fig. 3l). Based on the results, we conclude that long-term inhibition of CRBN induces mitochondrial oxidative stress and irreversible mitochondrial dysfunction.

\section{Long-term CRBN depletion reduces the viability of HepG2 and HeLa cells}

Mitochondrial permeability transition pore opening due to increased calcium uptake and excessive ROS production has been shown to disrupt mitochondrial respiration, leading to dysfunction and apoptosis $(23,24)$. Given that CRBN knockdown induced irreversible mitochondrial dysfunction and release of cytochrome $\mathrm{C}$, we further examined the effects of its depletion on apoptotic death. Cell viability and proliferation rate were significantly decreased in both CRBN-depleted (HepG2 and HeLa) cell lines (Fig. 4A, B; Supplementary Fig. 1D, E). Fluorescenceactivated cell sorting (FACS) analysis showed an increase in $\mathrm{G} 1$ and $\mathrm{G} 2 / \mathrm{M}$ phases and concomitant decrease in $\mathrm{S}$ phase in shCRBN cells (Fig. 4C). Furthermore, caspase3/7 activities were elevated in shCRBN cells (Fig. 4D). Western blot analysis revealed a marked increase in levels of apoptotic markers, including cleaved PARP, cleaved caspase 7, p53, and p21, in shCRBN cells (Fig. 4E). Long-term treatment with TD-165 reduced the viability of both HepG2 and HeLa cells (Fig. 4F, G). Notably, however, transient knockdown of CRBN via siRNA did not affect cell viability (Supplementary Fig. 4E). These results suggest that mitochondrial dysfunction due to long-term CRBN depletion promotes cell death.
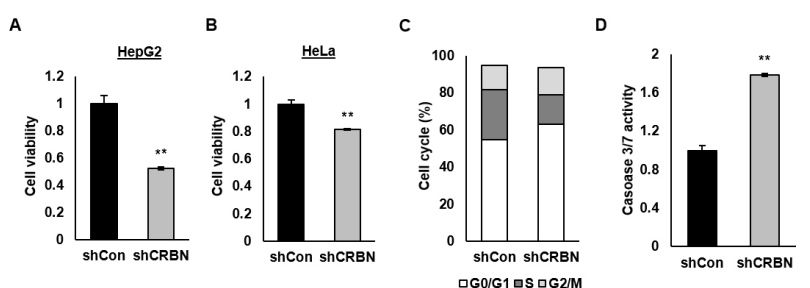

E

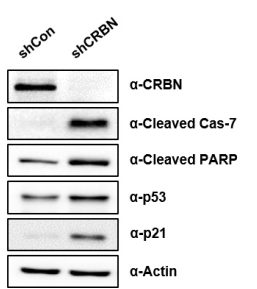
G
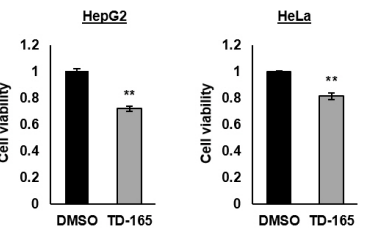

Fig. 4. Long-term depletion of CRBN promotes cancer cell death. (A, B) Effects of CRBN knockdown on viability of HepG2 and HeLa cells were determined with the Cell titer-Glo assay. (C) Flow cytometry analysis of cell cycle changes in shControl and shCRBN HepG2 cells. (D) Quantification of caspase 3/7 activity in shControl and shCRBN cells. (E) Western blot analysis of proteins related to apoptosis (cleaved caspase-7, cleaved PARP, p53 and p21). (F-G) Quantitative analysis of viability following TD-165 treatment for 12 days in (F) HepG2 and (G) HeLa cells. All results are presented as mean \pm S.D. of at least three independent experiments. P-values were calculated using unpaired student's t-test. ${ }^{*} \mathrm{P}<0.05$ and ${ }^{*} * \mathrm{P}<0.01$ vs control.

\section{DISCUSSION}

In this study, we demonstrated that CRBN knockdown leads to excess mitochondrial ROS production and impairment of mitochondrial respiration. In particular, long-term but not transient CRBN depletion led to accumulation of mitochondrial superoxide and mitochondrial dysfunction resulting in cell death. A recent investigation showed that upon activation of TLR4 signaling, CRBN suppresses mitochondrial ROS generation through inhibiting ubiquitination of ECSIT in monocytes (12). Considering that CRBN possesses the LON domain and associated protease activity in neuroblastoma cells (11), it is plausible that this region of CRBN is essential for mitochondrial homeostasis. Data from our CRBN knockdown experiments are consistent with the earlier finding that Lonp1 defects promote cellular senescence by inducing loss of mitochondrial integrity and mtDNA damage (9). Furthermore, given that upregulation of Lon protease is associated with aggressive cancer phenotypes, CRBN may serve as a potential therapeutic target (25).

CRBN interacts directly with AMP activated protein kinase (AMPK) and inhibits its catalytic activity $(26,27)$. AMPK, a wellknown regulator of mitochondrial ROS levels and energy homeostasis (28), is stimulated under multiple stress conditions, such as hypoxia and oxidative stress $(29,30)$, and plays a central role in maintaining mitochondrial biogenesis through regulation of mitochondrial fission, fusion, and mitophagy (29). Although we observed increased AMPK activity (data not shown) and decreased protein levels of motifusin-2 (MFN2), a key regulator of mitochondrial fusion (Fig. 3l), in shCRBN cells, staining analyses with autophagy markers revealed no significant differences between shControl and shCRBN cell lines (data not shown). Furthermore, the levels of two mitochondrial quality control proteins, PTEN-induced kinase 1 (PINK1) and Parkin $(3,31)$, remained unchanged in shCRBN cells relative to the control cell group (data not shown). Based on these results, it is unlikely that AMPK activation upon CRBN depletion contributes to mitochondrial dysfunction. Considering that CRBN is a substrate receptor of E3 ligases and also acts as a molecular chaperone, stabilized or immature substrates that are currently unknown may contribute to CRBN depletion-mediated mitochondrial dysfunction and subsequent apoptotic death in cancer cells.

\section{MATERIAIS AND METHODS}

\section{Cell cultures}

HEK293T (ATCC, CRL-11268), HepG2 (ATCC, HB-8065) and HeLa (ATCC, CCL-2) cells were cultured in Dulbecco's Modified Eagle Medium (Welgene, LM001-05) supplemented with 10\% fetal bovine serum (Gibco, 16000) and an antibiotic-antimycotic (Gibco, 15240) in a humidified atmosphere containing $5 \% \mathrm{CO}_{2}$ at $37^{\circ} \mathrm{C}$.

Transfection and establishment of stable cell lines

Cells were plated at $70-80 \%$ confluence and transfected with plasmid DNA using X-tremeGENE (Roche, 6366546001) or siRNA 
using Lipofectamine ${ }^{\text {TM }} 3000$ (ThermoFisher Scientific, L3000015) according to the manufacturers' protocols. The siCRBN sequence was as follows: sense, 5'-GAAGUUUACGGCCACCAAAdTdT-3', and antisense, 5'-UUUGGUGGCCGUAAACUUCdTdT-3'. HEK 293T cells were co-transfected with lentiviral plasmids expressing short hairpin RNA for CRBN (shCRBN) together with pSPAX2 and pMD2.G to produce viral particles. The shCRBN sequence used was 5'-GCTTGCAACTTGAATCTGATACTCGAGTATCAGA TTCAAGTTGCAAGCT-3'.

\section{Cell viability and caspase $3 / 7$ activity assays}

For measurement of cell viability and caspase $3 / 7$ activity, the CellTiter-Glo Luminescent Cell Viability assay (Promega, G7573) and Caspase-Glo ${ }^{\mathbb{R}}$ 3/7 assay system (Promega, G8091) were used, respectively, in accordance with the manufacturer's instructions. Luminescence intensity recorded using a multilabel plate reader (Perkin Elmer, Victor X3).

\section{Quantitative real-time PCR}

Total RNA was isolated from cultures using TRIzol reagent (Invitrogen, 15596026) according to the manufacturer's protocol. For quantitative real-time PCR (qRT-PCR) analysis of mRNA, synthesis and amplification of cDNAs was performed using the One-step SYBR Green PCR Master Mix (SFC, PGM5001) on a CFX96 Touch Real-Time PCR detection system (Bio-Rad). The beta-actin $(A C T B)$ primer sequences used were: (Forward, 5'CTGGAACGGTGAAGGTGACA-3', Reverse, 5'-AAGGGACTT CCTGTAACAATG-3') and CRBN primer sequences were: (Forward, 5'-AGTCTGCCGACATCACATAC-3', Reverse, 5'-GCACCA TACTGACTTCTTGAGG-3'). ACTB was employed as an endogenous normalization control. Each reaction was performed in duplicate and repeated in at least three different samples.

\section{Western blot analysis}

Cells were collected and lysed in RIPA buffer (Biosolution, BR002) containing Halt ${ }^{\mathrm{TM}}$ Protease Inhibitor Cocktail (Roche, 34044100). Proteins were separated using 7-15\% SDS-polyacrylamide gel electrophoresis and transferred to nitrocellulose membrane (GE Healthcare, 10600002). The following primary antibodies were used: CRBN (Atlas antibodies, HPA045910), Flag (Sigma-Aldrich, F3165), MFN2 (Abcam, ab50843), cytochrome C (Santa Cruz, sc13156), TOMM20 (Abcam, ab56783), alpha-tubulin (Sigma-Aldrich, T5168), cleaved caspase-7 (Cell signaling, \#9491), cleaved PARP (Cell signaling, \#9541L), p53 (Santa Cruz, sc126), p21 (Cell signaling, 2947S) and beta-actin (Santa Cruz, sc47778). Membranes were visualized using Western Pico or Femto ECL solution (ThermoFisher Scientific, 34580, 34096) on a chemiluminescent image analyzer (GE Healthcare, Al680).

\section{Measurement of intracellular ROS}

Intracellular ROS were detected using the oxidation-sensitive fluorescent probe dyes Dihydrorhodamine 123 (Invitrogen Molecular Probes, D23806) and MitoSOX Red (Invitrogen Molecular
Probes, M36008) in accordance with the manufacturer's instructions. Fluorescence intensity was measured using a fluorescence microplate reader (Perkin Elmer, Victor X3) at Ex/Em wavelengths of 488/525 nm for DHR123 and 510/580 nm for MitoSOX Red.

\section{Measurement of mitochondrial membrane potential}

For analysis of mitochondrial membrane potential, JC-1 probe (Molecular Probes, T3168) was used according to the manufacturer's instructions. Fluorescence images were visualized using laser scanning confocal microscopy (Zeiss, LSM810) or fluorescence microscope and JC-1 fluorescence quantitatively analyzed via flow cytometry (BD Biosciences, FACSVerse).

\section{Real-time ATP production and oxygen consumption}

To evaluate mitochondrial respiration, the real-time ATP rate assay kit (Agilent, 103591-100) was employed in accordance with the manufacturer's instructions. Real-time ATP production and oxygen consumption rate (OCR) were monitored with the aid of Seahorse XFp analyzer (Agilent). Cells at $90 \%$ confluency were plated on a XFp cell culture miniplate (Agilent, 1030250) in complete medium. The following day, cells were treated with XF DMEM, pH 7.4 (Agilent, 103575-100). Assay cycles involving 3 minutes of mixing and a 2-minute waiting period were conducted in triplicate. Following measurement of basal respiration, $1.5 \mu \mathrm{M}$ oligomycin (75351, Sigma-Aldrich) and $1 \mu \mathrm{M}$ rotenone/ antimycin A mix (R8875 and A8674, Sigma-Aldrich) were added and measured.

\section{Calcium assay}

Cytosolic intracellular calcium flux was measured with the FLIPR $^{\mathbb{R}}$ Calcium assay kit (Molecular Devices, R8191) in accordance with the manufacturer's instructions. Subsequently, fluorescence intensity was measured using a Flexstation ${ }^{\mathbb{R}}$ instrument (Molecular Devices) at Ex/Em wavelengths of 488/525 nm. Mitochondrial calcium flux was measured using Rhod2-AM fluorescent dye according to the manufacturer's instructions. Fluorescence images were visualized at Ex/Em wavelengths of $552 / 580 \mathrm{~nm}$ under a confocal microscope (Zeiss, LSM800).

\section{ACKNOWLEDGEMENTS}

This work was supported by a grant (CAP-15-11-KRICT) from the National Research Council of Science and Technology, Ministry of Science, ICT, and Future Planning, a grant (NRF2019M3E5D4069882) from the National Research Foundation, Ministry of Science and ICT and Future Planning, and a grant from the KRIBB Initiative Program.

\section{CONFLICTS OF INTEREST}

The authors have no conflicting interests. 


\section{REFERENCES}

1. Friedman JR and Nunnari J (2014) Mitochondrial form and function. Nature 505, 335-343

2. Hüttemann M, Lee I, Samavati L, Yu H and Doan JW (2007) Regulation of mitochondrial oxidative phosphorylation through cell signaling. Biochim Biophys Acta 1773, 17011720

3. Lim JW, Lee J and Pae AN (2020) Mitochondrial dysfunction and Alzheimer's disease: prospects for therapeutic intervention. BMB Rep 53, 47-55

4. Belhadj Slimen I, Najar T, Ghram A, Dabbebi H, Ben Mrad M and Abdrabbah M (2014) Reactive oxygen species, heat stress and oxidative-induced mitochondrial damage. A review. Int J Hyperthermia 30, 513-523

5. Shokolenko I, Venediktova N, Bochkareva A, Wilson GL and Alexeyev MF (2009) Oxidative stress induces degradation of mitochondrial DNA. Nucleic Acids Res 37, 25392548

6. Ott M, Gogvadze V, Orrenius S and Zhivotovsky B (2007) Mitochondria, oxidative stress and cell death. Apoptosis 12, 913-922

7. Ni H-M, Williams JA and Ding W-X (2015) Mitochondrial dynamics and mitochondrial quality control. Redox Biol 4, 6-13

8. Ishii T, Miyazawa M, Hartman PS and Ishii N (2011) Mitochondrial superoxide anion (O 2•-) inducible. BMB Rep 44, 298-305

9. Bota DA and Davies KJ (2016) Mitochondrial Lon protease in human disease and aging: including an etiologic classification of Lon-related diseases and disorders. Free Radix Biol Med 100, 188-198

10. Davalli P, Mitic T, Caporali A, Lauriola A and D'Arca D (2016) ROS, cell senescence, and novel molecular mechanisms in aging and age-related diseases. Oxid Med Cell Longev 2016, 3565127

11. Kataoka K, Asahi T and Sawamura N (2016) Mitochondrial cereblon functions as a Lon-type protease. Sci Rep 6, 29986

12. Kim MJ, Min Y, Shim J-H, Chun E and Lee K-Y (2019) CRBN is a negative regulator of bactericidal activity and autophagy activation through inhibiting the ubiquitination of ECSIT and BECN1. Front Immunol 10, 3389

13. Zhou L and Xu G (2019) Cereblon attenuates DNA damageinduced apoptosis by regulating the transcription-independent function of p53. Cell Death Dis 10, 1-13

14. Kamogashira T, Hayashi K, Fujimoto C, Iwasaki S and Yamasoba T (2017) Functionally and morphologically damaged mitochondria observed in auditory cells under senescenceinducing stress. NPJ Aging Mech Dis 3, 1-11

15. Gunter T, Buntinas L, Sparagna G, Eliseev R and Gunter K (2000) Mitochondrial calcium transport: mechanisms and functions. Cell calcium 28, 285-296

16. Görlach A, Bertram K, Hudecova $S$ and Krizanova O (2015) Calcium and ROS: a mutual interplay. Redox Biol 6, 260-271
17. Fonteriz RI, de la Fuente $\mathrm{S}$, Moreno A, Lobatón $\mathrm{CD}$, Montero M and Alvarez J (2010) Monitoring mitochondrial $[\mathrm{Ca} 2+]$ dynamics with rhod-2, ratiometric pericam and aequorin. Cell calcium 48, 61-69

18. Lazarowski ER and Boucher RC (2001) UTP as an extracellular signaling molecule. Physiology 16, 1-5

19. Kim K, Lee DH, Park S et al (2019) Disordered region of cereblon is required for efficient degradation by proteolysistargeting chimera. Sci Rep 9, 1-14

20. Yamamoto H, Itoh N, Kawano S et al (2011) Dual role of the receptor Tom20 in specificity and efficiency of protein import into mitochondria. Proc Natl Acad Sci U S A 108, 91-96

21. Miyazono Y, Hirashima S, Ishihara N, Kusukawa J, Nakamura K-i and Ohta K (2018) Uncoupled mitochondria quickly shorten along their long axis to form indented spheroids, instead of rings, in a fission-independent manner. Sci Rep 8, 1-14

22. Chen H, Detmer SA, Ewald AJ, Griffin EE, Fraser SE and Chan DC (2003) Mitofusins Mfn1 and Mfn2 coordinately regulate mitochondrial fusion and are essential for embryonic development. J Cell Biol 160, 189-200

23. Cao X, Zhao S, Liu D et al (2011) ROS-Ca2 + is associated with mitochondria permeability transition pore involved in surfactin-induced MCF-7 cells apoptosis. Chem Biol Ineract 190, 16-27

24. Baumgartner HK, Gerasimenko JV, Thorne C et al (2009) Calcium elevation in mitochondria is the main $\mathrm{Ca} 2+$ requirement for mitochondrial permeability transition pore (mPTP) opening. J Biol Chem 284, 20796-20803

25. Pinti $M$, Gibellini L, Liu $Y, X u$ S, Lu B and Cossarizza A (2015) Mitochondrial Lon protease at the crossroads of oxidative stress, ageing and cancer. Cell Mol Life Sci 72, 4807-4824

26. Lee KM, Yang S-J, Kim YD et al (2013) Disruption of the cereblon gene enhances hepatic AMPK activity and prevents high-fat diet-induced obesity and insulin resistance in mice. Diabetes 62, 1855-1864

27. Lee KM, Jo S, Kim H, Lee J and Park C-S (2011) Functional modulation of AMP-activated protein kinase by cereblon. Biochim Biophys Acta 1813, 448-455

28. Hardie DG, Ross FA and Hawley SA (2012) AMPK: a nutrient and energy sensor that maintains energy homeostasis. Nat Rev Mol Cell Biol 13, 251-262

29. Herzig S and Shaw RJ (2018) AMPK: guardian of metabolism and mitochondrial homeostasis. Nat Rev Mol Cell Biol 19,121

30. Mungai PT, Waypa GB, Jairaman A et al (2011) Hypoxia triggers AMPK activation through reactive oxygen speciesmediated activation of calcium release-activated calcium channels. Mol Cell Biol 31, 3531-3545

31. Vives-Bauza C, Zhou C, Huang Y et al (2010) PINK1-dependent recruitment of Parkin to mitochondria in mitophagy. Proc Natl Acad Sci U S A 107, 378-383 